\title{
GAS SLOSHING AND RADIO GALAXY DYNAMICS IN THE CORE OF THE 3C 449 GROUP
}

\author{
Dharam V. Lal ${ }^{1}$, Ralph P. Kraft ${ }^{1}$, Scott W. Randall ${ }^{1}$, William R. Forman ${ }^{1}$, Paul E. J. Nulsen ${ }^{1}$, Elke Roediger ${ }^{2}$, \\ John A. ZuHone ${ }^{3}$, Martin J. HardCastle ${ }^{4}$, Christine Jones ${ }^{1}$, and Judith H. Croston ${ }^{5}$ \\ ${ }^{1}$ Harvard-Smithsonian Center for Astrophysics, 60 Garden Street, Cambridge, MA 02138, USA \\ ${ }^{2}$ Jacobs University Bremen, P.O. Box 750 561, D-28725 Bremen, Germany \\ ${ }^{3}$ NASA-GSFC, 8800 Greenbelt Rd., Greenbelt, MD 20771, USA \\ ${ }^{4}$ University of Hertfordshire. School of Physics, Astronomy, and Mathematics, Hatfield AL 10 9AB, UK \\ ${ }^{5}$ University of Southampton, School of Physics and Astronomy, Southampton, SO17 1SJ, UK \\ Received 2012 July 24; accepted 2012 October 24; published 2013 January 29
}

\begin{abstract}
We present results from a $140 \mathrm{ks}$ Chandra/ACIS-S observation of the hot gas around the canonical FR I radio galaxy 3C 449. An earlier, shorter $30 \mathrm{ks}$ Chandra observation of the group gas showed an unusual entropy distribution and a surface brightness edge in the gas that could be a strong shock around the inner radio lobes. In our deeper data we find no evidence for a temperature increase inside of the brightness edge, but a temperature decrease across part of the edge. This suggests that the edge is a "sloshing" cold front due to a merger within the last $\lesssim 1.3-1.6$ Gyr. Both the northern and southern inner jets are bent slightly to the west in projection as they enter their respective lobes, suggesting that the sloshing core is moving to the east. The straight inner jet flares at approximately the position where it crosses the contact edge, suggesting that the jet is entraining and thermalizing some of the hot gas as it crosses the edge. We also detect filaments of X-ray emission around the southern inner radio jet and lobe which we attribute to low entropy entrained gas. The lobe flaring and gas entrainment were originally predicted in simulations of Loken et al. and are confirmed in our deep observation.
\end{abstract}

Key words: galaxies: individual (3C 449) - hydrodynamics - intergalactic medium - X-rays: galaxies: clusters

Online-only material: color figures

\section{INTRODUCTION}

Groups and clusters of galaxies in the local universe continuously undergo mergers with small groups and galaxies falling into the larger potential. Even if the mass ratio of the infalling object to that of the cluster is relatively small, the merging process can have a significant impact on the intracluster medium (ICM) of the larger body. In particular, a minor merger can offset the gas core from the center of the dark matter potential. The offset gas core will oscillate around the center of the dark matter potential creating a characteristic spiral pattern in the temperature and density structure (Ascasibar \& Markevitch 2006). This characteristic spiral pattern, referred to as "sloshing," is commonly seen in Chandra observations of groups (Machacek et al. 2011) and clusters of galaxies (Markevitch \& Vikhlinin 2007). The gas temperature and density change discontinuously at the sloshing front, but the pressure typically remains at or near equilibrium across the front. Hydrodynamic simulations of the process demonstrate that subsonic flows are created parallel to the sloshing fronts (Ascasibar \& Markevitch 2006).

Massive elliptical galaxies lie at the centers of every cluster of galaxies, and most or all of these galaxies contain supermassive black holes which have outbursts and drive jets. The understanding of astrophysical jets requires detailed knowledge of the hydrodynamic parameters of the material both of the collimated flow and of the external medium. The Chandra X-Ray Observatory (Chandra) has opened a new window into the study of extragalactic radio jets with its ability to spatially resolve $\mathrm{X}$-ray emission from the active nuclei, non-thermal emission from ultra-relativistic particles accelerated in the jets and lobes created at internal shocks in the jets, and thermal emission from the galaxy/group/cluster environments that confine the radioemitting plasma. To date, non-thermal X-ray emission from more than 100 extragalactic radio jets and hot spots has been reported (Massaro et al. 2011 and references therein), and the gaseous environments of several hundred additional radio galaxies spanning a wide range of both jet power and environmental richness have been studied in detail, e.g., M 87 (Forman et al. 2005, 2007), Hydra A (Nulsen et al. 2005), MS 0735.6+ 7421 (McNamara et al. 2005), Centaurus A (Kraft et al. 2007b; Hardcastle et al. 2003), 3C 33 (Kraft et al. 2007a), 3C 288 (Lal et al. 2010), among many others. The visible appearance of a radio galaxy depends in detail on the complex interplay between the jet power, the internal Mach number of the jet, the external gas pressure and pressure profile, and the velocity field in the external medium.

The two types of radio galaxies where the morphology is most influenced by the external medium are the narrow angle tailed (NAT) and the wide angle tailed (WAT) radio galaxies. NATs, such as 3C 129 (Lal \& Rao 2004), are radio galaxies in which the twin jets are sharply bent in the same direction and are believed to be the result of the infall of the jet host galaxy into a larger potential (Jaffe \& Perola 1973). WATs, on the other hand, such as 3C 465 (Hardcastle et al. 2005), typically reside in group and cluster cores and show more gentle bending of the radio jets. How this jet bending relates to gas motions in the external medium remains uncertain.

The radio source 3C 449 is a canonical example of a Fanaroff-Riley class I radio galaxy (Fanaroff \& Riley 1974) and exhibits a complex radio morphology on spatial scales from parsecs to hundreds of kiloparsecs. The source is characterized by an unresolved core, two symmetrically opposed jets, and very extended lobes (Perley et al. 1979). The jets are relativistic near their bases and decelerate significantly within $10^{\prime \prime}$ $(=3.3 \mathrm{kpc})$ from the core. Each jet is remarkably straight for $\sim 50^{\prime \prime}$ ( $\simeq 16.7 \mathrm{kpc}$ ) from the nucleus before deviating sharply toward the west, dropping in surface brightness and terminating in large diffuse lobes (Feretti et al. 1999). Since both jets bend in 
Table 1

Chandra Observations Analyzed in This Paper

\begin{tabular}{lcccc}
\hline \hline ObsID & Detector & ObsDate & $\begin{array}{c}\text { Exposure } \\
(\mathrm{ks})\end{array}$ & $\begin{array}{c}\text { Cleaned Exposure } \\
(\mathrm{ks})\end{array}$ \\
\hline 4057 & ACIS-S & 2003 Sep 18 & 29.6 & 29.18 \\
11737 & ACIS-S & 2010 Sep 14 & 53.0 & 52.35 \\
13123 & ACIS-S & 2010 Jun 20 & 60.7 & 59.92 \\
\hline
\end{tabular}

the same direction, rotation effects cannot cause this morphology (Birkinshaw et al. 1981). On larger scales, the outer lobes (Feretti et al. 1999) are diffuse and are relaxed in appearance, although the southern lobe is spherical while the northern lobe appears to be elongated.

The hot gaseous atmosphere around 3C 449 has been observed with ROSAT, XMM-Newton, and Chandra. ROSAT observations (Hardcastle et al. 1998) showed that the group gas is not spherically symmetric, and that southern lobe is embedded in a rim of hot gas, with a deficit at the position of the radio lobe suggesting that a cavity has been created by the inflation of the lobe. No X-ray emission was detected in the vicinity of the northern outer lobe, suggesting a significant external pressure difference between the northern and southern outer lobes. More recently, Croston et al. (2003), using XMM-Newton observations, measured the temperature and density profile of the ICM to a distance of $\sim 100 \mathrm{kpc}$ from the nucleus and argued that the outer lobes of 3C 449 are significantly older than expected from spectral age estimates if they are evolving buoyantly. They also showed that the gas temperature of the group gas was significantly higher than that predicted from the temperatureluminosity relationship. Interestingly, Croston et al. (2003) also found that the temperature decreased radially outward from the core, perhaps suggesting that the core had been heated by the nuclear outburst

Sun et al. (2009), using a $30 \mathrm{ks}$ archival Chandra observation, reported a significant peak in the entropy profile of the 3C 449 cluster gas, consistent with the temperature gradient found by Croston et al. (2003) and supporting their scenario of active galactic nucleus (AGN) heating. The level of heating and the unusual entropy profile are indicative of supersonic inflation of the radio lobes at fairly high Mach number, $M \sim 2$ (Sun et al. 2009). If the inflation of the inner lobes is driving a strong shock into the group gas then the current outburst is completely detached from the larger scale bubbles (i.e., that the outer lobes are no longer receiving energy from the nucleus and are evolving buoyantly). Re-examination of this archival Chandra observation indicates the presence of a surface brightness edge in the gas (at low statistical significance) at roughly the distance of the inner radio lobes. Shocks have been detected around the radio lobes of a number of radio galaxies, but most of these are relatively weak with $M<1.5$. Shock heating of the ICM is thought to play a key role in the energy budget of the gas cores of clusters and groups, and in the suppression of cooling flows. Shocks with $M>1.5$ are particularly important because they are relatively rare and the equivalent heat input due to the entropy increase in the gas is $\sim 10 \%$. Here, we present results from a new deep Chandra observation of the hot gas atmosphere around 3C 449 to better understand the dynamics of the radio lobe/ICM interaction.

This paper is organized as follows: Section 2 contains a summary of the observational details. An overview of the data and observed structures are presented in Section 3. Results of the data analysis are presented in Section 4, and we discuss their interpretation and implications in Section 5. Section 6 contains a brief summary and conclusions. A Wilkinson Microwave Anisotropy Probe cosmology with $H_{0}=73 \mathrm{~km} \mathrm{~s}^{-1} \mathrm{Mpc}^{-1}$, $\Omega_{M}=0.27$, and $\Omega_{\Lambda}=0.73$ is adopted (Spergel et al. 2007). At a redshift of $z=0.017085$ of the host galaxy of 3C 449, the lookback time is $2.26 \times 10^{8} \mathrm{yr}$, the angular size scale is $20.6 \mathrm{kpc}$ $\operatorname{arcmin}^{-1}$, and the luminosity distance, $D_{L}$ is $71.1 \mathrm{Mpc}$. All coordinates are $\mathrm{J} 2000$. The elemental abundances that we quote are relative to the solar value tabulated by Anders \& Grevesse (1989). Absorption by gas in our galaxy $\left(N_{\mathrm{H}}=1.19 \times 10^{21} \mathrm{~cm}^{-2}\right.$ Dickey \& Lockman 1990) is included in all our spectral fits. All spectral analysis errors are $90 \%$ confidence, while all other errors are $68 \%$ confidence. North is up and east is to the left in all images. Throughout the paper, we define the spectral index $\alpha$ in the sense that $S_{v} \propto v^{-\alpha}$.

\section{DATA PROCESSING}

3C 449 was observed with Chandra using ACIS-S. We summarize the dates, exposure times and Chandra ObsIDs in Table 1. The data were reprocessed starting with the level 1 event files and using CIAO version 4.3 with the up to date gain and calibration files applied (CalDB version 4.4.1). All observations were performed in Very Faint data mode. Observation specific bad pixel files were produced and applied and events flagged with $A S C A$ grades 1, 5, and 7 were excluded. Standard procedures were followed to exclude high background; i.e., for each observation, point sources and the region containing the brightest diffuse group emission were excluded, and a light curve was extracted in the $10-12 \mathrm{keV}$ range. We excluded time intervals when the count rate deviated by more than $20 \%$ from the mean. The cleaned exposure times for each ObsID are listed in Table 1 with the total cleaned exposure time being $141.5 \mathrm{ks}$.

\subsection{Background Modeling}

Since the 3C 449 group extends well beyond the Chandra field of view, it was necessary to use an external data set for background subtraction. For background subtraction during spectral and imaging analyses, we use the blank sky observations ${ }^{6}$ appropriate for the chip and epoch of the observation of interest. The background files were processed in the same manner as the observations, using the same background filtering, bad pixel and gain files, and were reprojected to match the observations. The source and background images were normalized in the 10-12 keV range. We checked for excess soft Galactic X-ray emission in the observations by extracting spectra from sourcefree regions far off-axis (away from the group emission) and compared to spectra extracted from the dark sky background files. We found no significant differences.

\subsection{Radio Data}

We use an archival Westerbork Synthesis Radio Telescope (WSRT) image of 3C 449 at $0.61 \mathrm{GHz}$ (Perley et al. 1979) taken from the online 3CRR Atlas $^{7}$ which provides well-calibrated, well-sampled images. We also make use of data from the Very Large Array (VLA) archive at 1.4, 4.9, and $8.4 \mathrm{GHz}$. Details regarding the radio observations are summarized in Table 1 of Feretti et al. (1999). The VLA data were reduced in

\footnotetext{
6 See http://cxc.harvard.edu/contrib/maxim/acisbg

7 ATLAS catalog: radio images and other data for the nearest 85 DRAGNs (radio galaxies and related objects) in the so-called 3CRR sample of Laing et al. (1983). Available at http://www.jb.man.ac.uk/atlas/index.html.
} 


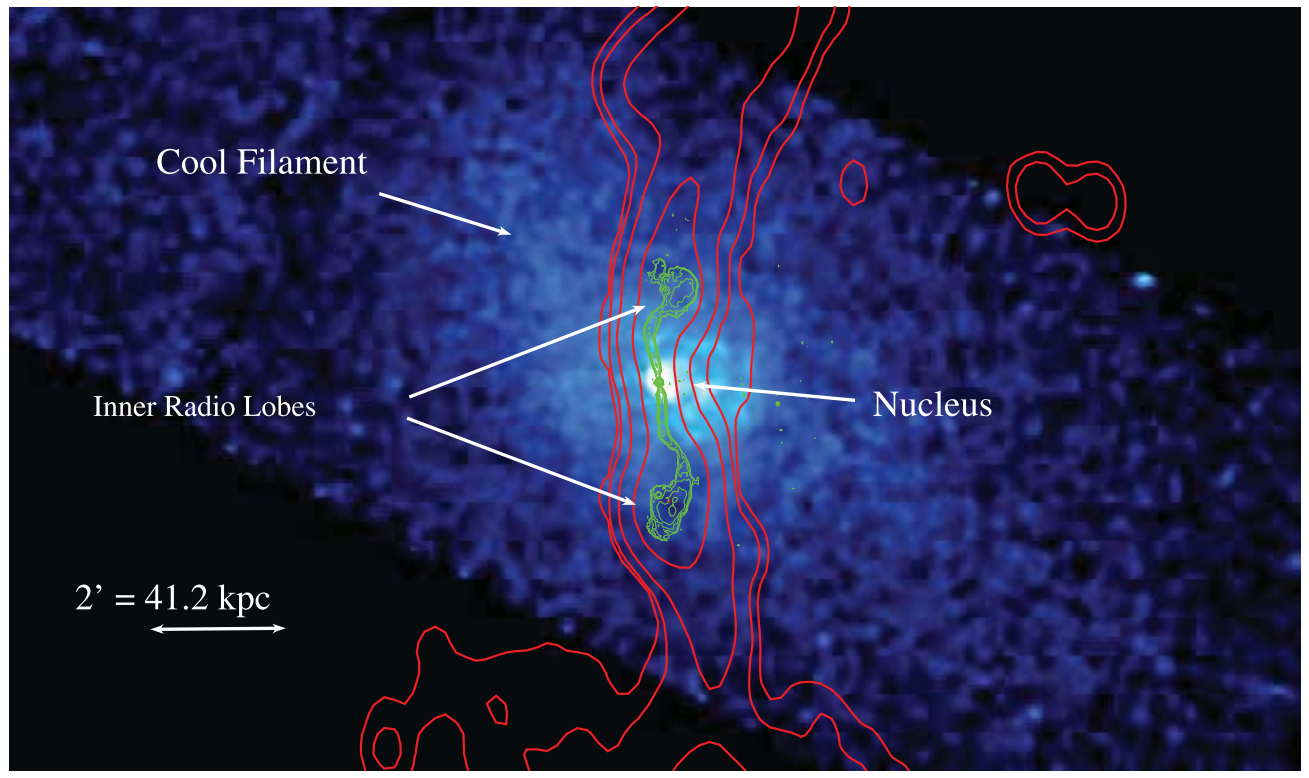

Figure 1. Combined Chandra/ACIS-S image of $3 \mathrm{C} 449$ in the $0.5-2.0 \mathrm{keV}$ band. The image is binned to $2^{\prime \prime}$ pixels and smoothed with a Gaussian $\left(\sigma=4^{\prime \prime}\right)$. All point sources, other than the active nucleus of $3 \mathrm{C} 449$, have been removed. We see diffuse thermal emission in the soft band extending over the entire field of view (12' $\sim 240 \mathrm{kpc}$ along the east-west axis). The green and red contours are taken from the 8.4 ( $7^{\prime \prime} .1$ Gaussian beam) and $0.6 \mathrm{GHz}$ ( $47^{\prime \prime} .9 \mathrm{Gaussian}$ beam) radio maps, respectively.

(A color version of this figure is available in the online journal.)

Astronomical Image Processing System following the standard procedures. Absolute flux density calibration was tied to the observations of a suitable flux calibrator close to the VLA archive data.

\section{OVERVIEW OF X-RAY DATA}

Figure 1 shows a Gaussian-smoothed, backgroundsubtracted, exposure corrected image of 3C 449 from the Chandra/ACIS-S observations in the $0.5-2.0 \mathrm{keV}$ band with point sources removed. Radio contours from the WSRT $0.61 \mathrm{GHz}$ map (red) and the VLA $8.4 \mathrm{GHz}$ map (green) are overlaid to show the relationship between the hot gas and the radio plasma. To enhance the visibility of structures in the diffuse emission, we created an additional zoom-in of the central region with features of interest labeled; this is shown in Figure 2. These two images highlight the following features:

1. There is an elongated diffuse structure extending to $\sim 330^{\prime \prime}$ ( $\simeq 110 \mathrm{kpc}$ ) to the northeast of the core, labeled "cool filament."

2. There are two arc-shaped tangential edges in the surface brightness $\sim 100^{\prime \prime}(\simeq 33 \mathrm{kpc})$ to the southeast and to the west of the core.

3. A close-up view of the jet in the southern direction shows the presence of a "tunnel-like" feature, marked with a dashed line. The tunnel connects the core and the southern radio lobe and is filled with the radio jet.

4. There is an X-ray cavity, i.e., a decrement in X-ray emission, at the location of the southern inner radio lobe. This cavity is surrounded by a rim of enhanced brightness. No cavity is detected at the position of the northern inner radio lobe.

In this paper we present a detailed spatial and spectral analysis of these features to constrain the gas dynamics of the system and better understand the interaction of the hot gas with the radio jets.

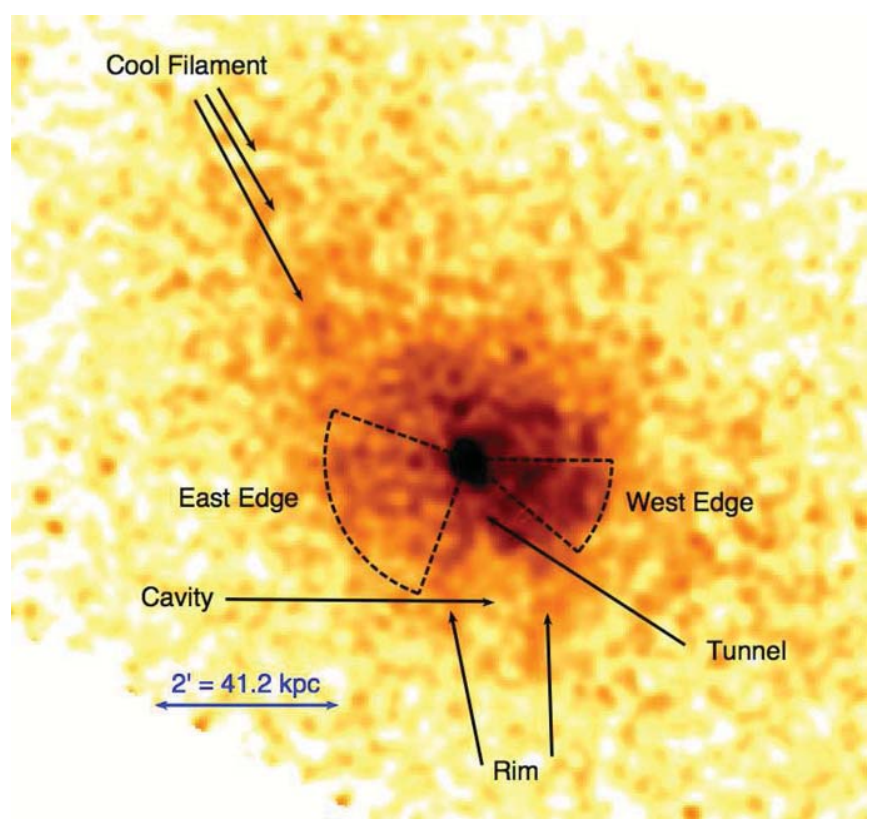

Figure 2. Background-subtracted, exposure-corrected, Gaussian-smoothed $\left(\sigma=4^{\prime \prime}\right)$ Chandra image in the energy range $0.5-2.0 \mathrm{keV}$. Features of interest that are discussed in the text are labeled including the cool filament, the eastern and western edges, the rims and cavity associated with the southern inner radio lobe, and the tunnel associated with the southern inner jet. The two wedges denote the regions for which the surface brightness profiles in Figures 9 and 10 were extracted

(A color version of this figure is available in the online journal.)

\section{DATA ANALYSIS}

\subsection{Large-scale Properties}

To characterize the large-scale spatial distribution of the $\mathrm{X}$-ray-emitting gas, we derived the radial surface-brightness profile of the ICM. The profile was fitted with an azimuthally 


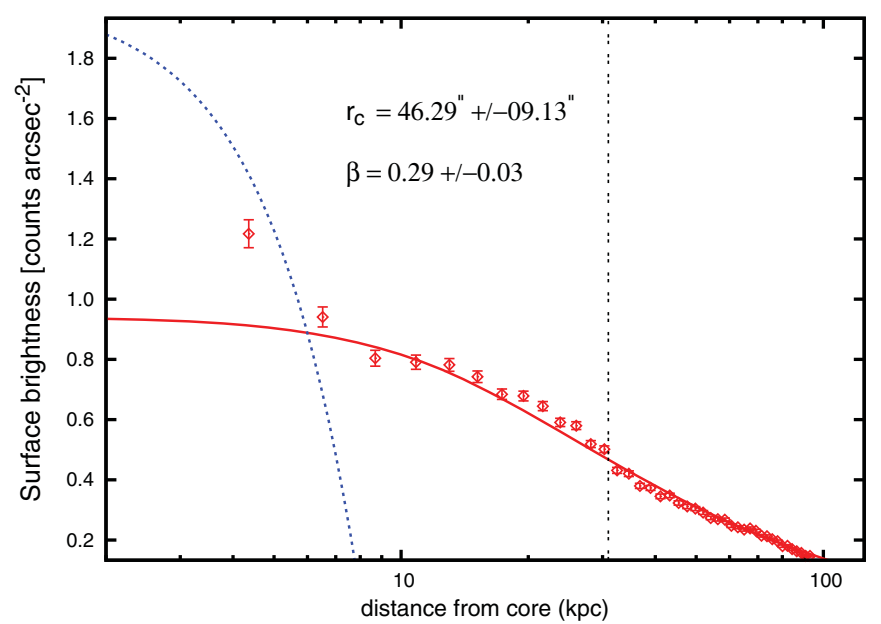

Figure 3. Azimuthally averaged $0.5-2.0 \mathrm{keV}$ radial surface brightness profile of 3C 449 group gas centered on the active nucleus created from the combined Chandra data. The model is the best-fitting point-source plus $\beta$-model, with $\beta=$ 0.29 and $r_{\mathrm{c}}=46.29(=15.41 \mathrm{kpc})$. The vertical line shows the approximate location of the surface brightness edge.

(A color version of this figure is available in the online journal.)

symmetric $\beta$-model shown in Figure 3. We found a best-fitting model with $\beta=0.29 \pm 0.03$, core radius $r_{c}=46$ '.29 \pm 9 '. 13 $(=15.41 \pm 3.03 \mathrm{kpc})$ with $\chi_{v}^{2}$ (degrees of freedom, dof $)=$ $1.32(35)$ (confidence ranges are $1 \sigma$ ). The core radius is roughly consistent within uncertainties with the best-fitting model of Croston et al. (2003) derived from the XMM-Newton observation, but the power-law index is somewhat flatter. The $X M M$ Newton data covered a much larger solid angle on the sky, and there are clearly non-azimuthally symmetric structures in the gas, so the exact fit parameters will depend on exactly what distances from the nuclei are fit. To emphasize the asymmetric structures revealed in Figures 1 and 2, we created a residual image by subtracting the average $\beta$-profile from the data. The residual image in Figure 4 shows that the northeast filament and an X-ray enhancement to the west of the nucleus are detected with high (>99\%) significance. The western edge of the enhancement to the west is the position of one of the surface brightness edges. A substructure 5'.6 (= $112 \mathrm{kpc})$ to the southwest is also visible.

\subsection{Large-scale Thermodynamic Structure}

We fitted a single temperature $\mathrm{VAPEC}^{8}$ model after excising point sources and the central AGN to the gas emission in the energy range $0.5-5.0 \mathrm{keV}$, with the absorption column density set at the Galactic value, $N_{\mathrm{H}}=1.19 \times 10^{21} \mathrm{~cm}^{-2}$ (Dickey \& Lockman 1990). The abundances for O, Fe, and $\mathrm{Si}$ were free to vary, with the rest of the elemental abundances (to which the spectral fits are insensitive) fixed at $0.5 \times$ solar and the temperature was free to vary. The goal of this large scale fit is to constrain the elemental abundances from a single spectral fit over a large region with a large number of counts. The single temperature model provided a good fit with $k T=1.66_{-0.05}^{+0.04} \mathrm{keV}$ for $\chi^{2}$ (dof) 0.98 (171), and the abundances for $\mathrm{O}, \mathrm{Fe}$, and Si were found to be $0.12_{-0.12}^{+0.21}, 0.32_{-0.06}^{+0.07}$, and $0.56_{-0.16}^{+0.17} \times$ solar, respectively. The subsolar value of the $\mathrm{Fe}$ and $\mathrm{Si}$ abundance, and the extremely low value of the $\mathrm{O}$ abundance, are consistent with X-ray observations of a sample of early-type galaxies (Humphrey \& Buote 2006). We use these values of the elemental

\footnotetext{
8 Astrophysical Plasmas Emission Code-http://cxc.harvard.edu/atomdb
}

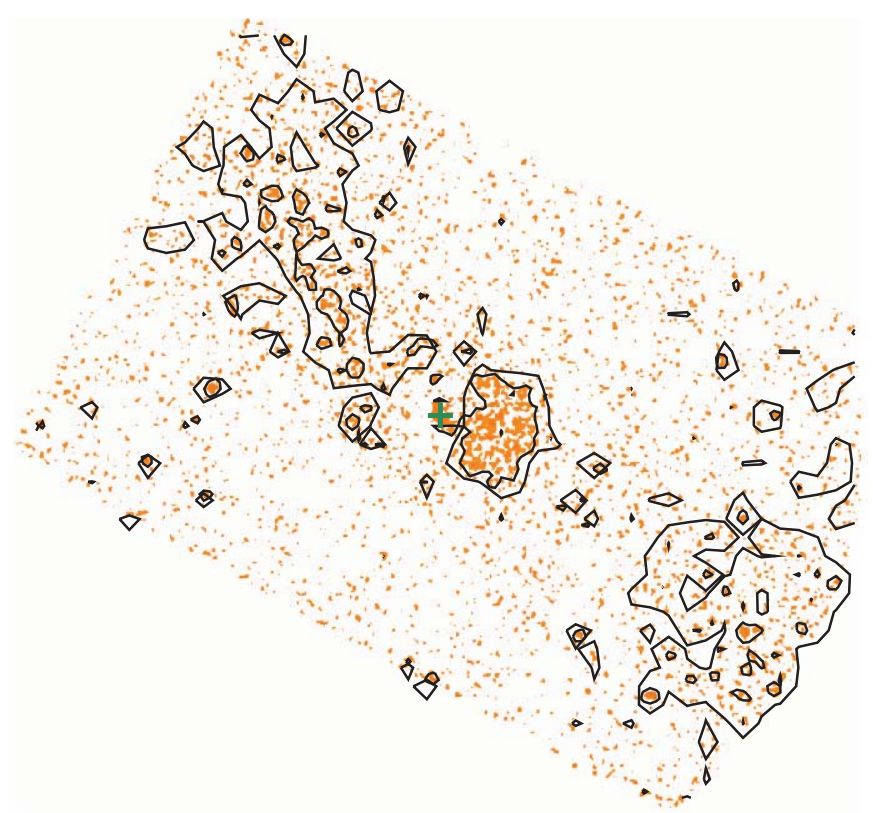

Figure 4. Residual image produced by subtracting an azimuthally symmetric $\beta$ model. This image is smoothed using a Gaussian kernel with radius of $3^{\prime \prime}$. The contours are placed at $1.22 \times 10^{-6}$ and $1.77 \times 10^{-7}$ counts s$^{-1} \operatorname{arcsec}^{-2}$. The cool filament and an asymmetric core emission are clearly seen. The green cross-point at the center shows the location of the radio/X-ray core.

(A color version of this figure is available in the online journal.)

abundance for all subsequent spectral fits and in the creation of the temperature maps.

We fit the temperature of the gas in 20 radial bins from $\sim 2 \mathrm{kpc}$ to $\sim 120 \mathrm{kpc}$. An analytical approximation to the best-fitting values of $k T(r)$ over the range of radii is given below:

$$
k T=1.64 \times\left[1+\left(\frac{r}{110}\right)^{2}\right]^{-0.8} \mathrm{keV} .
$$

This approximation is shown in Figure 5 by the black solid line. From this temperature profile, we also find that the temperature decreases with increasing radius, which is consistent with the results of Croston et al. (2003). The average temperature in the wedge containing the cool filament to the northeast (between the position angle, P.A., interval $10^{\circ}$ and $70^{\circ}$ ) shows a similar change but the overall temperature is lower than the azimuthally averaged temperature profile.

Using the temperature and surface brightness profile, we compute the electron density profile. We fit an analytic approximation to the best-fitting values of $n_{\mathrm{e}}(r)$

$$
n_{\mathrm{e}}=3.08 \times 10^{-3} \times\left[1+\left(\frac{r}{15.41}\right)^{2}\right]^{-\frac{3}{2} \times 0.29} \mathrm{~cm}^{-3}
$$

along with $k T(r)$ to obtain profiles of pressure and entropy, which are shown in Figure 6. The pressure profile shows a smooth decline as a function of distance from core; whereas the entropy profile shows a gradual increase to $10 \mathrm{kpc}$, followed by a rise to $80 \mathrm{kpc}$ (an entropy bump) and finally a flattening, which is consistent with the results of Sun et al. (2009).

\subsection{Temperature Maps}

We created temperature maps of the hot ICM. These maps were created using the method developed by O'Sullivan et al. (2005) and Maughan et al. (2006), which is outlined in full by 


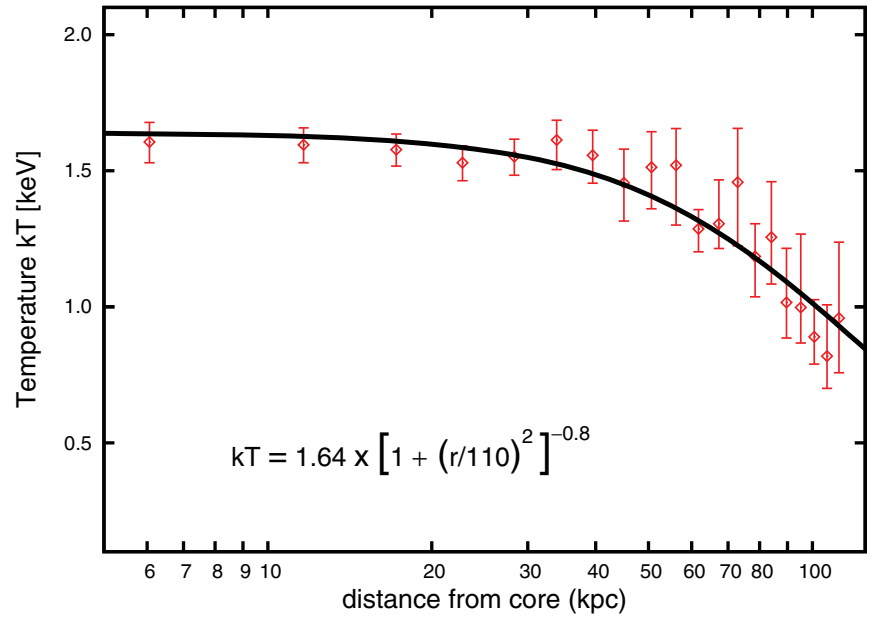

Figure 5. Plot of temperature variation in the environment of $3 \mathrm{C} 449$ as a function of distance from the core for azimuthally averaged profiles; the blackline is the best-fitting analytic $k T(r)$ model.

(A color version of this figure is available in the online journal.)

Randall et al. (2008). Briefly, after excluding the AGN and other point sources, the $0.5-5.0 \mathrm{keV} \mathrm{X}$-ray image was binned in $5^{\prime \prime} \times$ $5^{\prime \prime}$ pixels size and at the position of each pixel, source and background spectra are extracted in a circular region surrounding the pixel. The radius of this region at each pixel is defined adaptively so that the extracted region contains 1000 backgroundsubtracted counts. For each spectrum, a single VAPEC model was fitted in the energy range $0.5-5.0 \mathrm{keV}$, with the absorption column density set at the Galactic value. The abundance for $\mathrm{O}$, $\mathrm{Fe}$, and Si fixed to $0.12,0.32$, and 0.56 , respectively, the abundance for the remaining elements fixed to $0.5 \times$ solar, and the temperature allowed to vary. The temperature map is presented in the top panels of Figure 7 and the typical uncertainties in the temperatures are $\sim 10 \%$ at $90 \%$ confidence. Note that the formal statistical uncertainty in every pixel is roughly identical, but the pixels are not independent, so any small scale structures are not significant. The large scale (tens of arcsec) structures presented in different colors are significant, however.

The temperature maps reveal a rich diversity of patchy temperature structures, the most significant of which are a small cool core (shown as the blue at the center of the image) and the prominent cool $<1 \mathrm{keV}$ filament region. The latter is a wedge between P.A. $10^{\circ}$ and $35^{\circ}$, coincident with the asymmetry seen in the X-ray brightness, which we call the "cool filament."

We also generated projected pseudo-pressure and pseudoentropy maps, similar to those presented by Churazov et al. (2003) and Randall et al. (2008), shown in the bottom left and bottom right panels of Figure 7 , respectively. The column density map $n_{\text {col }}$ was estimated using the square root of the 1.2-2.5 keV Chandra image, as in Forman et al. (2007). Combining this with the temperature map $k T$, we compute the pseudo-pressure, $P$, and pseudo-entropy, $K$, at each pixel, given by $P=n_{\text {col }} k T_{\text {pix }}$, and $K=k T_{\text {pix }} n_{\text {col }}^{-2 / 3}$, respectively. Unlike temperature maps, the symmetric pressure distribution, which peaks at the X-ray surface brightness peak, indicates the hydrostatic nature of 3C 449. The pressure map shows no hint of sharp changes at the locations of the radio lobes. The pressure map, which is near hydrostatic equilibrium, is consistent with simulations of sloshing galaxy cluster cores, where the pressure distribution stays the same during the sloshing (ZuHone et al. 2010). The structure in pseudo-entropy is similar to what we see

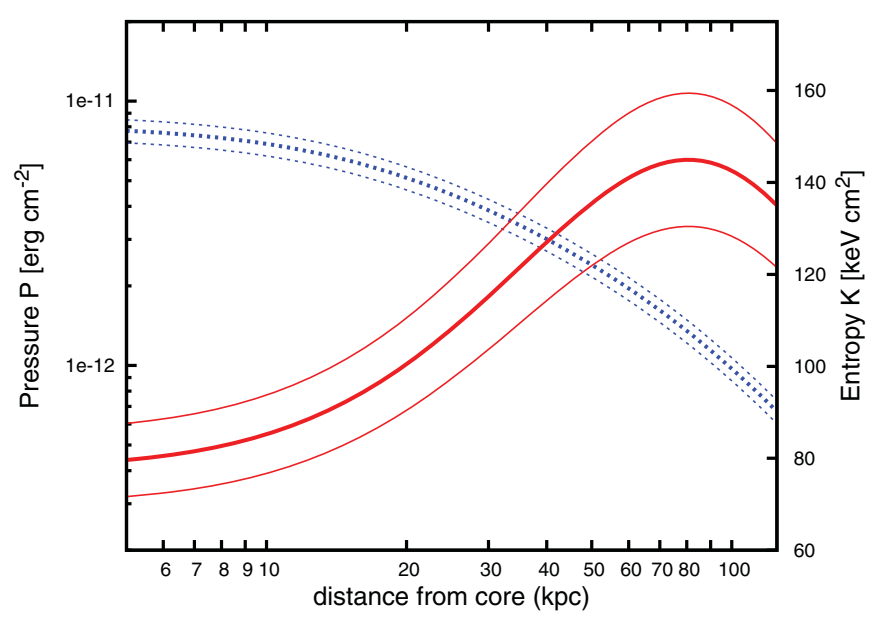

Figure 6. Azimuthally averaged radial pressure (left axis: blue points) and entropy (right axis: red lines) profiles for 3C 449. The uncertainties on pressure and entropy are at $\sim 90 \%$ confidence.

(A color version of this figure is available in the online journal.)

in the temperature map; i.e., the pseudo-entropy map shows a small low-entropy core, and a low-entropy tail to the northeast, which is coincident with the cool filament/tail.

This method produces a temperature map with correlated pixels (O'Sullivan et al. 2005; Maughan et al. 2006), and the scale over which the pixels are correlated is not clear from the temperature map alone. Therefore, as a check we produced a second map using a number of independent spectral regions from the background-subtracted, exposure-corrected Chandra image and residual image. In particular, we considered several wedges based on the Chandra $0.5-2.0 \mathrm{keV}$ surface brightness images (Figures 1, 2, and 4) and the thermodynamic image (Figure 7, top panel images). We further divided each wedge, named from (A) to (I) into several elliptical annuli $(1,2, \ldots$, $n$, where annulus " 1 " is close to the core and " $n$ " is farthest from the core) to investigate temperature structure. Spectra for these annular quadrants were fitted with the VAPEC model. The abundance was again fixed to $0.12,0.32,0.56 \times$ solar for $\mathrm{O}, \mathrm{Fe}$, and $\mathrm{Si}$, respectively, and $0.5 \times$ solar for rest of the elements. The confidence ranges for regions with net counts $\gtrsim 3000$ are small, $0.04-0.07 \mathrm{keV}$ (90\% confidence, $\chi^{2} 0.98-1.58$ for 88-239 dof), whereas for regions with low net counts $(\lesssim 1000)$ the confidence ranges are large, $0.13-0.45 \mathrm{keV}(90 \%$ confidence, $\chi^{2} 0.58-1.62$ for $18-239$ dof). A crude temperature map (in which all the bins are independent) along with our spectral extraction regions are shown in Figure 8. This crude temperature map with independent pixels confirms the general features seen in the adaptive map including the cool filament to the northeast and the lack of any large temperature jumps associated with other features in the gas.

\subsection{Surface Brightness Edges}

We find two edges in the surface brightness distribution, one 97.9 (= $32.6 \mathrm{kpc}$ ) southeast of the nucleus and another 96.7 (= $32.2 \mathrm{kpc})$ to the west, as marked in Figure 2. The presence of these edges in the surface brightness distribution implies a sharp change in the gas density or temperature (or both) of the gas across the edges. Chandra has observed a large number of similar features in other clusters, such as A1795 (Markevitch et al. 2001), A3667 (Vikhlinin et al. 2001), M 87 (Forman et al. 2005, 2007), Hydra A (Nulsen et al. 2005), 

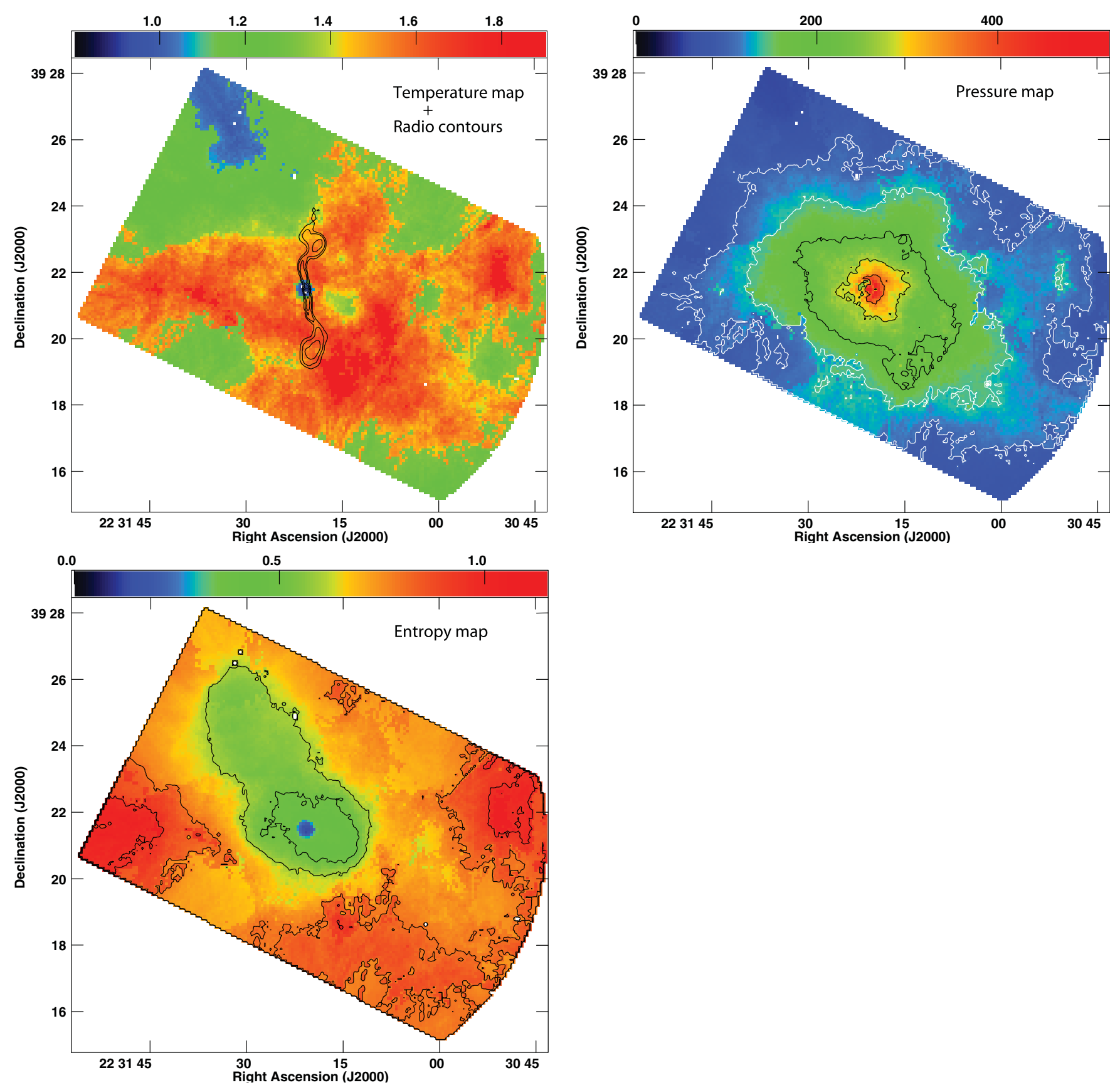

Figure 7. Thermodynamic maps of the 3C 449 group gas derived from the Chandra data using the technique described in Randall et al. (2008). Top left map has radio contours overlaid on the temperature map, the top right map is the pseudo-pressure map, and bottom left map is the pseudo-entropy map. The color bar scale gives the temperature in $\mathrm{keV}$ in the temperature map and is in arbitrary units in the pressure and entropy maps.

(A color version of this figure is available in the online journal.)

MS 0735.6 + 7421 (McNamara et al. 2005), A1201 (Owers et al. 2009), and 3C 288 (Lal et al. 2010), and they are generally attributed to three phenomena: merger cold fronts, sloshing of cluster cores, and shocks due to nuclear outbursts (Markevitch \& Vikhlinin 2007). To investigate whether the observed surface brightness decrease is associated with shocks or cold fronts, we extracted surface brightness profiles and spectra in several regions of interest. Following the analysis of Markevitch \& Vikhlinin (2007), we determined the temperature and pressure across the edge to evaluate which of the three scenarios; merger cold-front, sloshing cold-front, or supersonic inflation of radio lobes, is most plausible.
We fitted VAPEC models to annuli in two sectors (marked in Figure 2) centered on 3C 449. The vertex of the annuli was fixed at the nucleus, but their radii were adjusted to so that three annuli were interior and two annuli were exterior to the edge. The goal of this spectral fitting was to determine whether the gas temperature interior to the edges was hotter or cooler than the exterior gas temperature. Only the temperatures and normalizations were free parameters in these fits. Plots of the surface brightness and temperature profiles for the two different sectors as a function of radius from the active nucleus are shown in panels (a) and (c) of Figures 9 and 10, respectively. For the southeastern wedge, we measured temperatures of $1.61_{-0.07}^{+0.06} \mathrm{keV}$ 


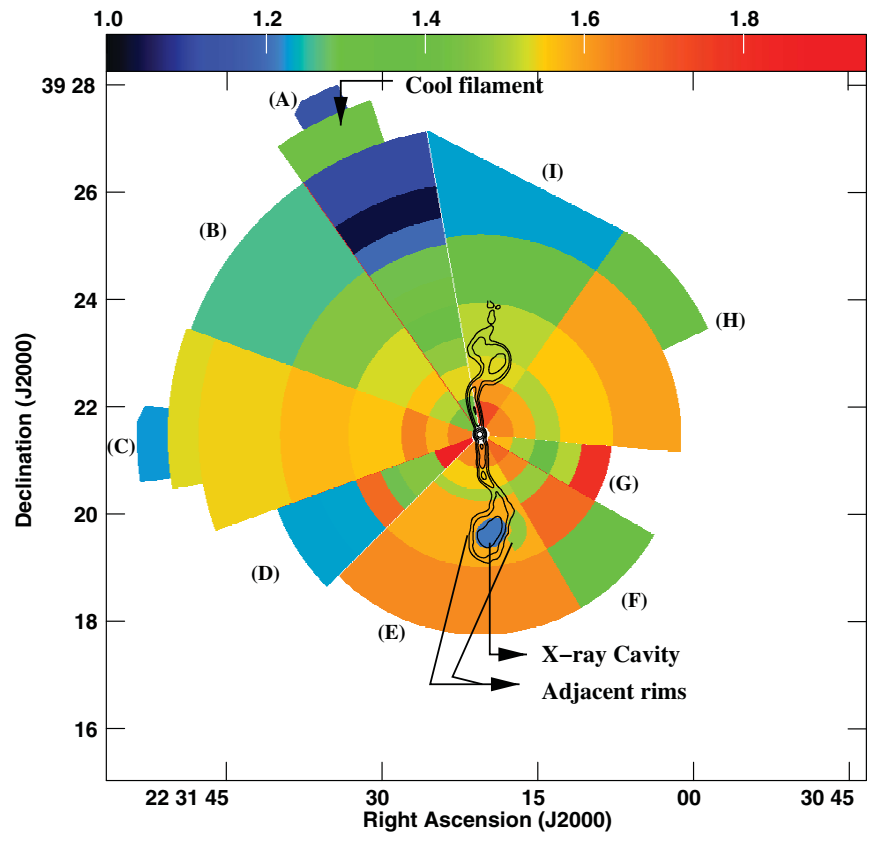

Figure 8. Projected temperature map using the merged data. Point sources are excluded. The color bar gives the temperature scale in $\mathrm{keV}$. The confidence ranges for regions with net counts $\gtrsim 3000$ are low, $0.04-0.07 \mathrm{keV}$ (inner sectors, close to the core), whereas for regions with low net counts $\lesssim 1000$ are high, $0.13-0.45 \mathrm{keV}$ (outer sectors) at the $90 \%$ confidence level.

(A color version of this figure is available in the online journal.)

inside the jump and $1.51_{-0.06}^{+0.07} \mathrm{keV}$ outside the jump. For the western wedge, we measured temperatures of $1.35_{-0.03}^{+0.09} \mathrm{keV}$ inside the jump and $1.86_{-0.23}^{+0.18} \mathrm{keV}$ outside the jump. The temperature ratios for the southeastern and western edges are $1.02_{-0.07}^{+0.06}$ and $0.72_{-0.09}^{+0.08}$, respectively. The uncertainties are at $90 \%$ confidence.

We fitted the surface brightness across the edge in the sectors shown in Figure 2 in the energy range $0.5-2.0 \mathrm{keV}$ with a broken power-law density model to determine the density and pressure jumps across the edges. To minimize projection effects, ideally one would prefer to measure the deprojected temperature profile across the edges; however, the limited number of photons and small temperature differences do not permit this and we use the projected temperatures measured above. The lack of large temperature variation suggests that projection effects are not large. The deprojected density and pressure profiles as a function of distance from the cluster center are shown in Figures 9 and 10, panels (b) and (d), respectively. Both brightness profiles have a characteristic shape corresponding to an apparent abrupt jump in the gas density. Best-fit radial density models consisting of two power laws with a jump are shown in panel (b), and the projected model surface brightness profiles are overlaid on the data in panel (a) of Figures 9 and 10. From the amplitude of the best fitting surface brightness model, we derived a density jump of $1.23_{-0.18}^{+0.20}$ for the southeastern edge and $1.89_{-0.21}^{+0.18}$ for the western edge. The confidence ranges for the density jumps were computed from the extremes of the $90 \%$ confidence ranges for the best fitting surface brightness model. We assumed spherical symmetry in the deprojection of the surface brightness to derive the densities.

The X-ray morphology of the filaments around the inner southern radio lobe is similar to features seen in nearby radio galaxies. These structures are formed when AGN jets push into the local ICM, evacuating cavities, and often creating bright rims of X-ray emission from the displaced gas. In more evolved remnant cavities, the rims tend to be cooler and more dense than the nearby ambient ICM, as in A2052 (Blanton et al. 2003) and Perseus (Fabian et al. 2006), whereas in more recent outbursts they often show higher temperatures associated with shocks, as in NGC 4552 (Machacek et al. 2006), Hercules A (Nulsen et al. 2005), Centaurus A (Croston et al. 2009), and NGC 5813 (Randall et al. 2011). To test for a temperature difference in the rims, we extracted spectra from the southern bubble and a region on the rim just outside the bubble, subtracted a local background region, and fit each with an absorbed VAPEC model with the abundance fixed (see Section 4.2). We find best-fitting temperatures of $k T_{\mathrm{E}-\mathrm{rim}}=1.59_{-0.25}^{+0.41}$ for the east rim, $k T_{\mathrm{W}-\text { rim }}=$ $1.48_{-0.22}^{+0.32}$ for the west rim and $k T_{\text {cavity }}=1.21_{-0.16}^{+0.15}$ for the cavity, inside the rim. The (projected) temperature of the gas in the rims is consistent within uncertainties with that of the ambient gas. We conclude that these rims are most likely low entropy gas that has been uplifted by the radio bubbles and not shocks.

Finally, there is a clear decrease in X-ray surface brightness at the position of the southern inner jet. We labeled this as the "tunnel" in Figure 2. To test the significance of the tunnel-like feature, we extracted the total count rate in evenly spaced bins across the radio jet shown in Figure 2 (left panel). Figure 11 shows two distinct peaks adjacent to a clearly visible valley above the nominal surface brightness. If we take an average count rate from the highest three bins in each peak and compare it with the average count rate from the lowest three bins in the valley, we find that the gap is significant at about the $1.9 \sigma$ level. It seems that the gas may be compressed or the gas may have been uplifted to create a "sheath-like" feature, and the tunnel is filled with the relativistic plasma. Assuming the radius of the radio jet to be equal to the width of the tunnel as seen on the sky, we estimate the decrement in surface brightness due to the evacuated tunnel. That is, we assumed that the volume occupied by the jet is devoid of gas and that the jet is in the plane of the sky. We integrated the square of the density profile at the position of the jet to compute the surface brightness decrement created by the jet. The predicted decrement in emission measure is $\sim 0.86$, which is consistent with the measured decrement in emission measure of $0.75 \pm 0.09$ within $90 \%$ confidence ranges. This also suggests that the southern jet lies at or near the plane of the sky (i.e., the decrement would be smaller if the jet were far from the plane), which is consistent with the generally symmetric appearance of the inner jets.

\section{DISCUSSION}

We report the detection of several previously unknown features in the hot gas atmosphere around 3C 449. Both the surface brightness and temperature distributions of the gas display a complex, asymmetric morphology within $\sim 100 \mathrm{kpc}$ of the radio/X-ray core. Some features arise from the AGN activity, but others are better explained by an earlier group merger.

The first of the AGN-related features is a cavity at the position of the southern radio lobe which is surrounded by a rim of enhanced X-ray emission. The temperature of the rim is marginally higher than in the cavity, but is consistent with that of the ambient gas within the large error bars. Furthermore, there is a tunnel-like feature in the X-ray emission connecting the southern cavity and the group core (see Figure 11), which coincides with the southern radio jet. The radio jet seems to have created the tunnel, either by compressing the gas or by uplifting the gas to form a sheath-like feature. If the 

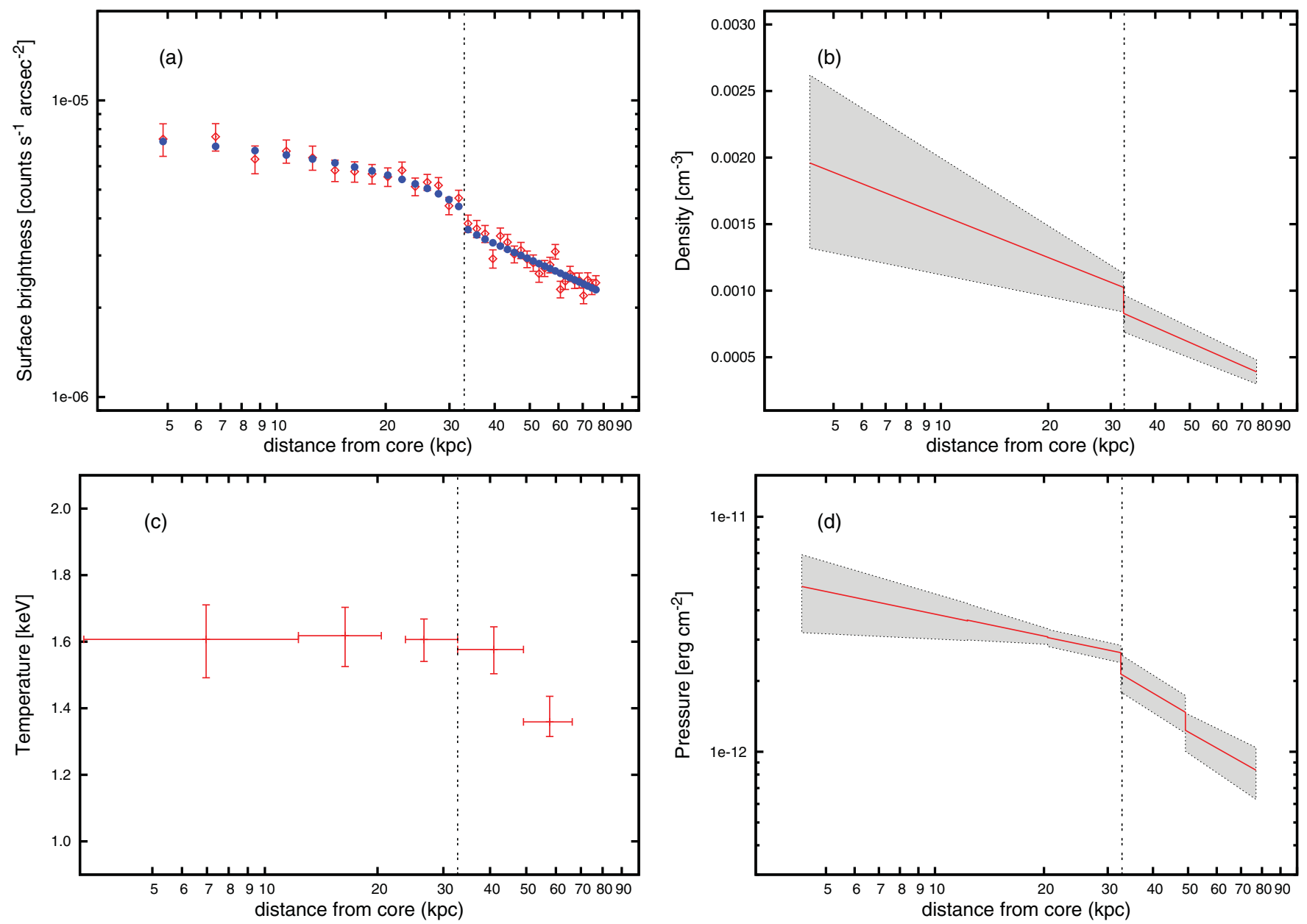

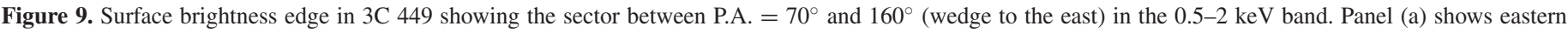

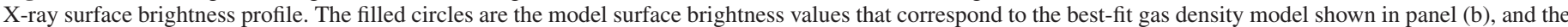

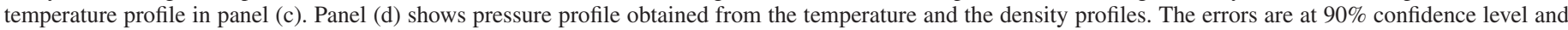
the vertical dashed line in each panel shows the position of the density jump.

(A color version of this figure is available in the online journal.)

former is true, the compressed gas should have been hotter than the surrounding gas and very short-lived since it would be overpressurized, whereas in the latter case the uplifted gas should have been cooler than the surrounding gas and in rough pressure equilibrium. It is unlikely that the jet is greatly overpressurized relative to the ambient gas, so the filaments surrounding the tunnel are likely to be low entropy gas that has been uplifted by the jet.

There are two arc-shaped surface brightness discontinuities in the gas to the southeast and to the west of the nucleus, at approximately $\sim 33.4 \mathrm{kpc}$ from the group core. Surface brightness edges as seen in the gas of 3C 449 are commonly attributed to three phenomena: remnant cold-core merger fronts, sloshing fronts, or supersonic inflation of radio lobes. In the presence of the obvious AGN activity in this group one might attribute these brightness edges to an AGN-driven shock, but there are several reasons why this is probably not the case. First, we find no evidence for a temperature increase of the gas interior to the edges as would be expected for a shock (see Figure 10 of Forman et al. (2007)). On the contrary, the gas interior of the western edge seems cooler than the exterior gas, typical of a cold front, and there is little temperature jump across the eastern edge. Even though the constraints on the temperature are not sufficient to rule out a weak $(M<1.5)$ shock, there is no evidence for a significant pressure jump either, which again favors an interpretation as cold fronts. Second, the AGN-driven shock is expected to enclose the cavities and radio lobes, whereas in 3C 449 the southern inner lobe is outside the discontinuity assuming even a small $\left(\sim 15^{\circ}\right)$ angle of the jet to the plane of the sky. It is hard to see how the lobes could be driving a shock if they are outside the shock. Third, the radio morphology does not support the shock interpretation. Instead of appearing as a momentum-dominated FR-II-type source like Cyg A (Wilson et al. 2006) or an overpressurized energy-driven bubble like Cen A (Kraft et al. 2003), both inner radio lobes are bent and wispy, suggesting a more gentle, nearly quasi-static interaction with the ambient gas. Fourth, the distance between the edge and the nucleus is larger in the $\mathrm{E} / \mathrm{W}$ axis than the $\mathrm{N} / \mathrm{S}$ axis where the lobes are located. Given that the radius of the lobes is only slightly larger than the jet, the shocks should either surround the lobe if the lobe is inflating supersonically or should form an oval around the jet/lobe pair if the jet is momentum dominated. If the edge is in fact a density/temperature discontinuity created by an AGN-driven shock, it is hard to see how the shock could propagate faster perpendicular to the axis of the driving piston than parallel to this axis. There are thus multiple lines of evidence to suggest that this edge is not an AGN-driven shock. 

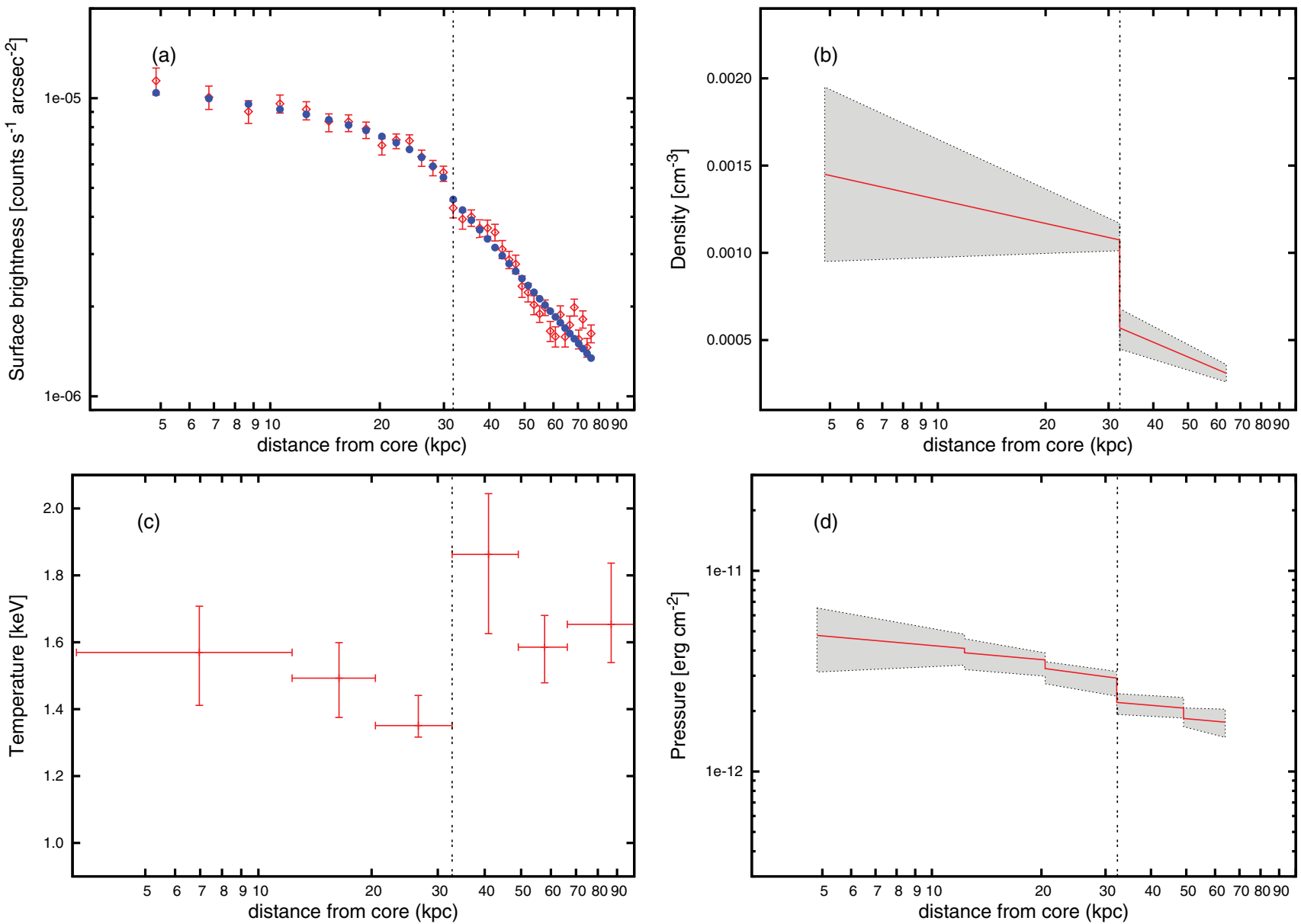

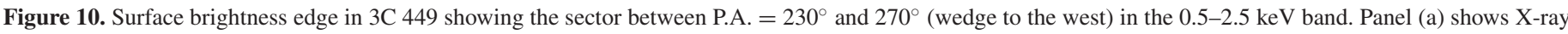

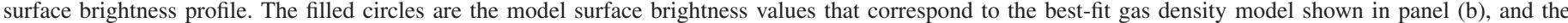

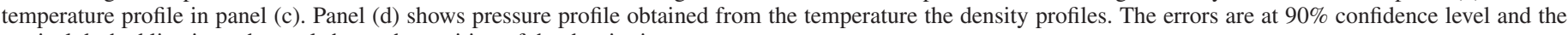
vertical dashed line in each panel shows the position of the density jump.

(A color version of this figure is available in the online journal.)

Even if the edges are not AGN-driven shocks, there does, however, appear to be a clear connection between the gas structure and the AGN because both inner jets bend eastward and expand to form the inner radio bubbles at the same group-centric radius where the edges are found. We discuss this connection below.

\subsection{Merger Dynamics}

\subsubsection{Remnant Merger Core}

We argued that the brightness edges in $3 \mathrm{C} 449$ are unlikely to be an AGN-driven shock, but more closely resemble cold fronts. A remnant or merger cold front is the interface between the dense cool gas core of a subcluster or subgroup and the hotter ambient gas the subgroup is moving through. Here, the cold fronts surround the group core itself and could imply a motion of the group core moving through the ambient group gas. Approximating the cool gas core as a blunt body, (Vikhlinin et al. 2001; Landau \& Lifschitz 1959), the pressure ratio between the pressure at the stagnation point and in the free stream region depends on the Mach number of the moving body. The conservative confidence intervals of pressure ratio are $1.25_{-0.23}^{+0.20}$ and $1.35_{-0.21}^{+0.23}$ for the southeast and west surface brightness edges, respectively. These correspond to a subsonic motion with Mach numbers, $M$, for the southeast and west edge of $0.53_{-0.23}^{+0.20}$ and $0.62_{-0.17}^{+0.18}$ (Figure 6 of Vikhlinin et al. 2001).

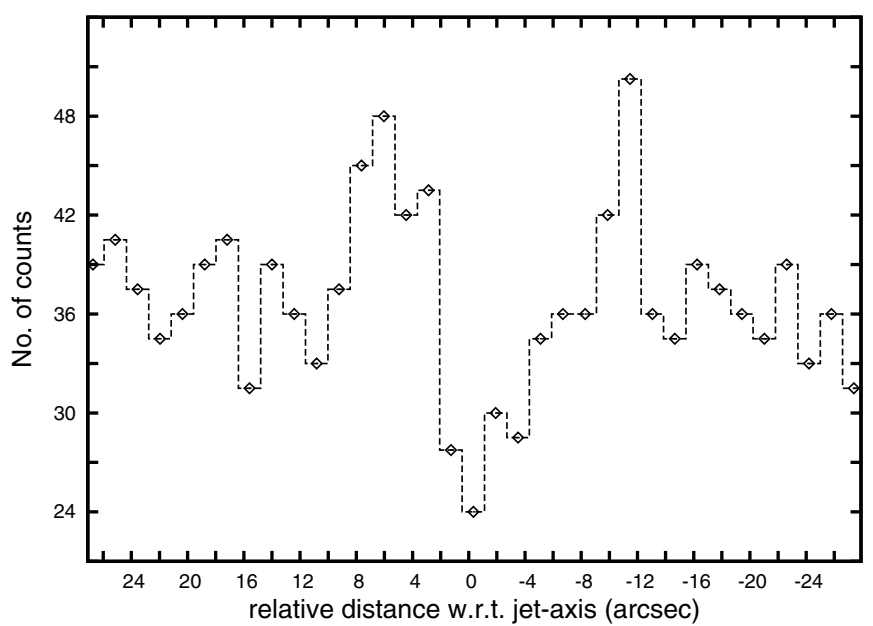

Figure 11. Histogram showing distribution of the counts in the energy range $0.5-2.0 \mathrm{keV}$ in evenly spaced binned regions taken across the "dashed" tunnellike feature (shown in Figure 2) in the shape of a box ( $56 . ' 6 \times 29$ '. 5 and centered at $\alpha=22: 31: 20.289, \delta=39: 20: 58.51$ ), which is $\sim 31^{\prime \prime}$ south of the nucleus.

The implied velocity in physical units are $354.3_{-150.2}^{+130.3} \mathrm{~km} \mathrm{~s}^{-1}$ and $414.8_{-113.0}^{+119.6} \mathrm{~km} \mathrm{~s}^{-1}$, respectively, where we used the gas temperature $k T=1.66_{-0.05}^{+0.04} \mathrm{keV}, \gamma=5 / 3$ and the mean molecular weight of the intracluster plasma $\mu=0.6$. The 

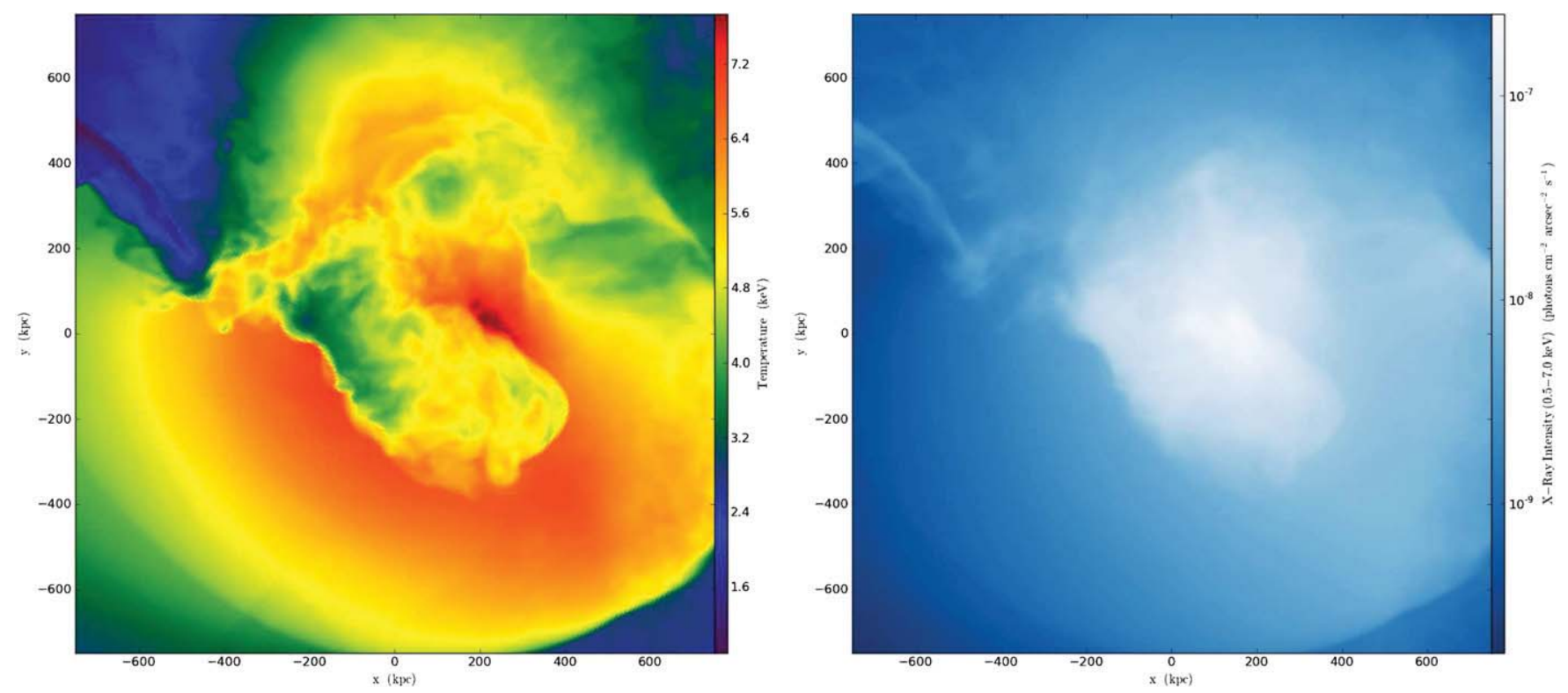

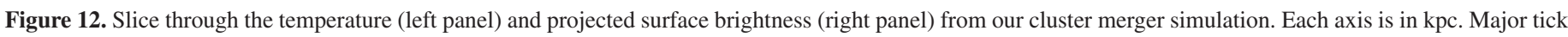

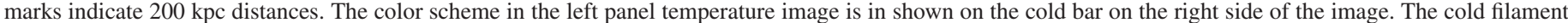

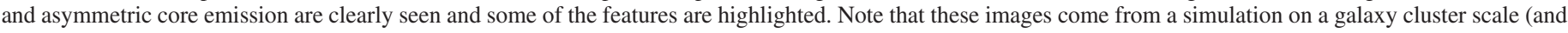

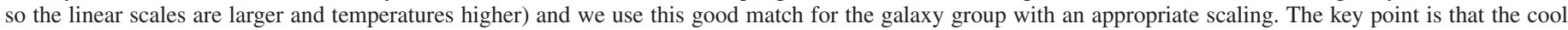
filament is a standard feature of late stage cluster mergers. Note that the sloshing cold front can be seen at the bottom right of both images.

(A color version of this figure is available in the online journal.)

presence of two edges roughly $180^{\circ}$ apart strongly argues against these features being the result of a remnant core. In addition, the relatively low (i.e., subsonic) velocity is not consistent with a remnant merger core at the center of the group potential. A remnant core that has infallen to the center of the group should be supersonic. Thus, we reject the remnant core scenario.

\subsubsection{Sloshing}

Sloshing cold fronts arise when an initially close to hydrostatic cluster or group atmosphere is slightly offset from its equilibrium position, e.g., by a minor or distant merger. The offset gas oscillates inside the central potential well, which leads to the formation of arc-like cold fronts wrapped around the cluster core (e.g., Ascasibar \& Markevitch 2006; Roediger et al. 2011; ZuHone et al. 2010). All but straight head-on mergers transfer angular momentum, and the sloshing takes on a spiral-like appearance in the orbital plane. If the line of sight (LOS) is perpendicular to the orbital plane, also the cold fronts form a typical spiral structure. For LOSs parallel to the orbital plane, the cold fronts are arranged as staggered arcs on opposite sides of the cluster.

The arrangement of the cold fronts in the 3C 449 group neither matches perfectly the cold remnant nor the pure sloshing scenario. Remnants typically have a surface brightness discontinuity on only one side, not two. At the group center, a remnant core should be moving supersonically with a clear pressure discontinuity indicative of its infall velocity and perhaps a bow shock (although the shocks are often not visible due to projection or limited signal-to-noise ratio of the data). Sloshing cold fronts on opposite sides of the core usually appear at different cluster-centric distances. If the southeastern and western cold front form a continuous sloshing front around the south of the group core, we expect another front at a different distance to the north, but there is no indication for such a brightness discontinuity in the data. The observed cold filament is explained by neither scenario.
However, all these features can be explained if the 3C 449 group is in a late stage of a major merger. Figure 12 displays a mock X-ray surface brightness and temperature map of a binary cluster merger with a mass ratio of $1: 3$ and with a large impact parameter (see Figure 8, $t=4.0$ Gyr epoch of ZuHone 2011, for entropy map of this simulation). The smaller subcluster or subgroup has had its entire gaseous atmosphere ram pressure stripped. This stripped gas forms a long cool tail or filament pointing away from the major group center. Due to the large impact parameter, the core of the larger group is not destroyed, but indeed sloshing motions are triggered. For low mass ratios and also at late stages the sloshing is not as well-ordered as in a minor merger, but more irregular, and the spiral or staggered arc pattern is distorted or missing. Scaling timescales and spatial scales of the cluster merger in Figure 12 to a group merger appropriate for the 3C 449 group, we estimate that the core passage occurred $\sim 1.3-1.6$ Gyr ago.

We emphasize that in this interpretation the surface brightness discontinuities we see in our X-ray image are the secondary, inner, sloshing cold fronts, not the primary ones typically seen in X-ray images. We note that in the residual surface brightness image in Figure 4, there is an excess of emission $\sim 115 \mathrm{kpc}$ to the southwest of the nucleus. This excess is at roughly the position/distance of the primary sloshing cold fronts (assuming that the surface brightness edges we see are in fact the secondary sloshing cold front), and may denote the position of the primary front or some larger scale asymmetry. There is no evidence of the primary sloshing edge in our data if we extend the profiles out to this distance, so whether it actually marks the position of the primary cold front is unclear. Sloshing can also introduce asymmetric features in the gas on large scales since the core (both gas and dark matter) of the group is offset from the center of the large scale gravitational potential. Finally we mention that the inflation of the large scale radio lobes has also input significant thermal energy into the gas and may well have distorted the appearance of the primary sloshing cold front. 
This interpretation could be evaluated with a deep Chandra mosaic.

The jet axis in 3C 449 is close to the plane of the sky. If the orbital plane of the merger was also close to the plane of the sky, it would have introduced bulk flows in this plane. However, the outer radio jets are very straight out beyond $100 \mathrm{kpc}$, which may imply a significant inclination between the merger plane and the plane of the sky.

\subsection{Radio Galaxy Dynamics}

Hydrodynamic simulations of jets crossing density discontinuities with shear flow show that well collimated jets can become partially disrupted (Loken et al. 1995), and the disruption of the jets of 3C 449 by this type of edge was first predicted by Katz-Stone \& Rudnick (1997). Assuming a moderate angle between the jets and the plane of the sky, it is plausible that the interface between the inner jets and inner lobes occurs exactly at the surface brightness edges seen in our data. If this is the case, the jets must be inclined $\sim 15^{\circ}$ relative to the plane of the sky. This angle is consistent with the angle of the radio-jet to the LOS, $\sim 75^{\circ}$ estimated via relativistic motion of the inner $(100 \mathrm{pc})$ jet (Feretti et al. 1999). Interestingly, one striking aspect of this radio source is the mirror symmetry of the jets and outer lobes about an axis through the central nucleus. The fact that both of the inner lobes of 3C 449 are bent in the same direction to the west suggests that the gas exterior to the edge is pushing them in that direction. This is entirely consistent with the sloshing picture described above. Since the gas is moving with the central galaxy and gas interior to the sloshing cold front, the jets interior to the edge remain straight and well collimated. Once the jet crosses the edge, however, it is partially disrupted, entrains group gas, and inflates to form inner radio lobes. Additionally, the simulations of (Loken et al. 1995) also show that this disruption can drag cold gas from the interior out beyond the edge, and we associate the filaments around the southern inner bubble with this process.

The displaced gas from the tunnel at the position of the southern inner jet has clearly been deposited around it to create a sheath-like feature, and the tunnel is filled with the radio-jet plasma. To our knowledge this is the first case where we detect the X-ray sheath enveloping an established and stable radio plasma jet. The X-ray enhancements around the tunnel must be low entropy gas that has been entrained and uplifted by the jet. If it were shock-heated gas, it would expand rapidly into the interstellar medium and would be invisible. The jet may be entraining material and developing an internal sheath in addition to creating the observed cocoon of X-ray emitting gas. This internal sheath may be the steeper spectral index component surrounding the jet reported by Katz-Stone \& Rudnick (1997). The entrainment of a small amount of thermal gas may therefore have a dramatic effect on the relativistic electron population and/or on the efficiency of particle acceleration.

We can estimate the work done on the X-ray emitting gas by the inflation of the inner bubbles. Assuming a spherical geometry for the southern bubble (with radius of $\simeq 13 \mathrm{kpc}$, located at a distance from the core of $1.9 \simeq 39 \mathrm{kpc}$ ), we find an average electron density of $1.1 \times 10^{-3} \mathrm{~cm}^{-3}$ and pressure of $5.4 \times 10^{-11} \mathrm{erg} \mathrm{cm}^{-3}$. The work required us to inflate the cavity against this pressure is $p V \simeq 1.46 \times 10^{57} \mathrm{erg}$, where $V$ is the volume of the cavity. Including the internal energy of the cavity, the total energy required to create both cavities, i.e., its enthalpy, would be $4 p V$ if it is filled with relativistic gas, amounting to about $1.2 \times 10^{58} \mathrm{erg}$. Furthermore, the age of the cavity can be estimated as the time required for the cavity to rise the projected distance from the radio core to its present location at the speed of sound, or buoyantly at the terminal velocity (Bîrzan et al. 2004), $7 \times 10^{7} \mathrm{yr}$. The mechanical power of the current outburst inflating the inner lobes is, $L_{\text {mech }}=2 \times 4 \mathrm{pV} /$ age $_{\text {cavity, }}$, relatively modest $\sim 5.7 \times 10^{42} \mathrm{erg} \mathrm{s}^{-1}$. We note that this only gives a lower limit to the jet power since the flow of plasma may continue into the outer lobes.

Finally we mention that the unusual entropy profile of the group gas cannot be explained by the supersonic inflation of the inner radio lobes. These inner lobes do not appear to be inflating supersonically, the entropy peak lies beyond the position of the inner lobes, there is clear evidence that this group is in the late stages of a merger, and the larger scale lobes are also probably evolving buoyantly. Hydrodynamic simulations of sloshing show transient oscillations of the entropy profiles (Roediger et al. 2011). We therefore attribute this unusual structure in the entropy profile to merging, not nuclear activity. Interestingly, the other group studied by Sun et al. (2009) that has an unusual entropy peak is UGC 2755. This group also hosts a powerful radio galaxy, and examination of the archival Chandra data on this object also shows features in the $\mathrm{X}$-ray gas on scales larger than the radio outburst indicative of sloshing/merging.

\section{CONCLUSIONS}

In this paper we presented an analysis of the hot gas around the canonical FR I radio galaxy 3C 449 based on 140 ks Chandra $\mathrm{X}$-ray data and archival radio data. The combined Chandra images reveals not only complex, asymmetric morphologies of surface brightness and temperature but an asymmetric core emission, sharp edges, a "tunnel-like" feature and a cavity associated with the southern inner jet and lobe, respectively. An earlier, shorter Chandra $30 \mathrm{ks}$ observation of the group gas showed an unusual entropy distribution and evidence for a surface brightness edge in the gas. We find in our deeper dataset that this edge is probably a sloshing cold front due to merger $\lesssim 1.3-1.6$ Gyr ago.

The straight inner part of the jet flares at approximately the position where it crosses the contact discontinuity, suggesting that the jet is entraining and thermalizing some of the hot gas as it crosses the edge. The lobe flaring and gas entrainment were originally predicted in simulations of Loken et al. (1995). It is not unreasonable, then, to interpret the fact that jets crossing density edges can become partially disrupted and inflate to form inner radio lobes as a consequence of observed signatures of interactions between an AGN and its surrounding medium. The tunnel and the filaments around the lobe demonstrate that the jets do not simply propagate losslessly through the ICM, but that even a small amount of swept up gas is not sufficient to completely disrupt the jets. In fact, entrainment of external material may play a central role in the evolution of jets from nucleus to hot spot/termination. Future deeper Chandra observations of this group would facilitate a more detailed study of the sloshing interface and of the entrained material along the southern inner jet and lobe.

D.V.L. thanks R. Johnson for many fruitful conversations and is grateful to N. P. Lee for repeated help with the "crude" temperature map. Support for this work was provided by the National Aeronautics and Space Administration through Chandra Award Number GO9-9111X issued by the Chandra X-Ray Observatory 
Center, which is operated by the Smithsonian Astrophysical Observatory for and on behalf of the National Aeronautics Space Administration under contract NAS8-03060. J.H.C. acknowledges support from the South-East Physics Network (SEPNet). This research has made use of software provided by the Chandra X-Ray Center in the application packages CIAO and Sherpa. This research has made use of the NASA/IPAC Extragalactic Database (NED) which is operated by the Jet Propulsion Laboratory, California Institute of Technology, under contract with NASA. This research has made use of NASA's Astrophysics Data System.

\section{REFERENCES}

Anders, E., \& Grevesse, N. 1989, GeCoA, 53, 197

Ascasibar, Y., \& Markevitch, M. 2006, ApJ, 650, 102

Birkinshaw, M., Laing, R. A., \& Peacock, J. A. 1981, MNRAS, 197, 253

Bîrzan, L., Rafferty, D. A., McNamara, B. R., Wise, M. W., \& Nulsen, P. E. J. 2004, ApJ, 607, 800

Blanton, E. L., Sarazin, C. L., \& McNamara, B. R. 2003, ApJ, 585, 227

Churazov, E., Forman, W., Jones, C., \& Böhringer, H. 2003, ApJ, 590, 225

Croston, J. A., Hardcastle, M. J., Birkinshaw, M., \& Worrall, D. M. 2003, MNRAS, 346, 1041

Croston, J. A., Kraft, R. P., Hardcastle, M. J., et al. 2009, MNRAS, 395, 1999

Dickey, J. M., \& Lockman, F. J. 1990, ARA\&A, 28, 215

Fabian, A. C., Sanders, J. S., Taylor, G. B., et al. 2006, MNRAS, 366, 417

Fanaroff, B. L., \& Riley, J. M. 1974, MNRAS, 167, P31

Feretti, L., Perley, R., Giovannini, G., \& Andernach, H. 1999, A\&A, 341, 29

Forman, W. R., Jones, C., Churazov, E., et al. 2007, ApJ, 665, 1057

Forman, W. R., Nulsen, P., Heinz, S., et al. 2005, ApJ, 635, 894

Hardcastle, M. J., Worrall, D. M., \& Birkinshaw, M. 1998, MNRAS, 296, 1098

Hardcastle, M. J., Worrall, D. M., Kraft, R. P., et al. 2003, ApJ, 593, 169
Hardcastle, M. J., Sakelliou, I., \& Worrall, D. M. 2005, MNRAS, 359, 1007

Humphrey, P. J., \& Buote, D. A. 2006, ApJ, 639, 136

Jaffe, W. J., \& Perola, G. C. 1973, A\&A, 26, 423

Katz-Stone, D. M., \& Rudnick, L. 1997, ApJ, 488, 146

Kraft, R. P., Birkinshaw, M., Hardcastle, M. J., et al. 2007a, ApJ, 659, 1008

Kraft, R. P., Nulsen, P. E. J., Birkinshaw, M., et al. 2007b, ApJ, 665, 1129

Kraft, R. P., Vázquez, S. E., Forman, W. R., et al. 2003, ApJ, 592, 129

Laing, R. A., Riley, J. M., \& Longair, M. S. 1983, MNRAS, 204, L151

Lal, D. V., Kraft, R. P., Forman, W. R., et al. 2010, ApJ, 722, 1735

Lal, D. V., \& Rao, A. P. 2004, A\&A, 420, 491

Landau, L. D., \& Lifschitz, E. M. 1959, Fluid Mechanics (London: Pergamon)

Loken, C., Roettiger, K., Burns, J. O., \& Norman, M. 1995, ApJ, 445, 80

Machacek, M., Nulsen, P. E. J., Jones, C., \& Forman, W. R. 2006, ApJ, 648,947

Machacek, M., Jerius, D., Kraft, R., et al. 2011, ApJ, 743, 15

Markevitch, M., \& Vikhlinin, A. 2007, PhR, 443, 1

Markevitch, M., Vikhlinin, A., \& Mazzotta, P. 2001, ApJL, 562, L153

Maughan, B. J., Ellis, S. C., Jones, L. R., et al. 2006, ApJ, 640, 219

Massaro, F., Harris, D., \& Cheung, C. C. 2011, ApJS, 197, 24

McNamara, B. R., Nulsen, P. E. J., Wise, M. W., et al. 2005, Natur, 433, 35

Nulsen, P. E. J., McNamara, B. R., Wise, M. W., \& David, L. P. 2005, ApJ, 628,629

O’Sullivan, E., Vrtilek, J. M., Kempner, J. C., David, L. P., \& Houck, J. C. 2005, MNRAS, 357, 1134

Owers, M. S., Nulsen, P. E. J., Couch, W. J., \& Markevitch, M. 2009, ApJ, 704, 1349

Perley, R., Willis, A. G., \& Scott, J. S. 1979, Natur, 281, 437

Randall, S., Forman, W. R., Giacintucci, S., et al. 2011, ApJ, 726, 86

Randall, S., Nulsen, P. E. J., Forman, W. R., et al. 2008, ApJ, 688, 208

Roediger, E., Brüggen, M., Simionescu, A., et al. 2011, MNRAS, 413, 2057

Spergel, D. N., Bean, R., Doré, O., et al. 2007, ApJS, 170, 377

Sun, M., Voit, G. M., Donahue, M., et al. 2009, ApJ, 693, 1142

Vikhlinin, A., Markevitch, M., \& Murray, S. S. 2001, ApJ, 551, 160

Wilson, A. S., Smith, D. A., \& Young, A. J. 2006, ApJL, 644, L9

ZuHone, J. A. 2011, ApJ, 728, 54

ZuHone, J. A., Markevitch, M., \& Johnson, R. E. 2010, ApJ, 717, 908 